\title{
Prediction of Cell Phone Client's Location utilizing Semantic Trajectory
}

\author{
J. Venkata Subramanian, S. Govindarajan
}

\begin{abstract}
Investigation on anticipating developments of cell phone consumers has pulled in a great deal of considerations lately. Considerable foreseeing procedures are created dependent on geographic zonal highlights of cell phone abuser's directions. In this research, we put forward a new methodology for anticipating the subsequent place of a client's development dependent in cooperation of the territory and semantic highlights of clients' directions. Center thought of the expectation structure depends on new cluster centered forecast technique it assesses the subsequent place a cell phone client dependent on the continuous practices of comparable clients in analogous group controlled by dissecting clients' normal conduct in semantic directions. Through an exhaustive assessment by tests, our proposition is appeared to convey fantastic execution.
\end{abstract}

Keywords: Semantic Trajectory, location prediction, spatial temporal, Reality Mining dataset, mobile phone, LBS

\section{INTRODUCTION}

In the recent years, a rapid growth of location based services such as map-reading examinations, network traffic administration and location based promotion. Because of the requirements of viable advertising and effective framework tasks, it is helpful for these Location Based Services to have the option to conjecture the exercises a client may carry out at the next location to visit. Along these lines, powerful and successful mobile location forecasting strategies for Location Based Services focusing on cell phone abusers are alluring.

As of late another type of location foreseeing strategies, named general-pattern based forecast, have risen. These forecasting strategies typically utilize the successive regular practices of mobile client's intelligence from assortments of cell phone clients' Global Positioning System directions, to anticipate the next movement of a mobile client. A few instances of the Global Positioning System direction, it ordinarily comprises of a succession of spatio temporal focuses. Along with the general-pattern[1] based forecast strategies, cell phone sequential pattern mining procedures are broadly utilized for breaking down patterns in cell phone client movement informational collections. In any case, they

Revised Manuscript Received on February 05, 2020.

* Correspondence Author

J. Venkata Subramanian*, Department of Computer Applications, SRM Institute of Science and Technology, Chennai, India, jvenkatmail@gmail.com

Dr. S. Govindarajan, Professor, Department of EDP, SRM Institute of Science and Technology, Chennai, India, govindan@srmist.edu.in

(C) The Authors. Published by Blue Eyes Intelligence Engineering and Sciences Publication (BEIESP). This is an open access article under the CC BY-NC-ND license (http://creativecommons.org/licenses/by-nc-nd/4.0/) will in general foresee mainstream areas where the vast majority visited, prompting the imbalanced information issue. Also, pattern based forecasting strategies for the most part make a forecast in particular if a foreseen development is fully tie through the precede of an outline, prompting forfeiture of review happening forecasting strategies.

Despite the fact that the disputes of finding cell phone clients' regular patterns in their directions have been examined in the writing, existing investigations for the most part consider just on the

geographic highlights of client directions. Observe that a geographic direction regularly comprises of an arrangement of geographic focuses labeled with timestamps. Accordingly, the continuous pattern of mobile client development conduct dependent on geographic direction is obliged by the geographic possessions of the direction information. For instance, the geographic separation and appearance somewhere in the range of Trajectory 1 and Trajectory 2 is nearer and more comparative than that somewhere in the range of Trajectory1 and Trajectory3. In this manner, some location forecast systems would anticipate the goal of Trajectory1 dependent on its geological closeness to Trajectory2. Moreover, such forecast techniques just deliberate recently hang around areas in addition here after not function admirably once formerly uncalled areas remain assured. Here we contend that only utilizing geographic data to anticipate the goal of a direction or a client's next location isn't adequate.

Semantic direction has been proposed by Alvares et al [1]. Essentially, a semantic direction comprises of a grouping of areas named with semantic labels to catch the milestones passed by. Those semantic marks of zones propose the activities being finished toward the path.

To help area forecast dependent on the semantic directions of cell phone clients, we propose a new location forecasting structure, named Semantic Predict, to assess the mobile user's next location movement. The structure comprises of 2 significant sectors they are disconnected data mining part and connected forecast part. In disconnected data mining part, embrace the thought of visit areas speak about mobile clients' crusade conduct. Toward get out the semantic [1] component since personage mobile clients crusade conduct, excavate the semantic direction patterns for every personage mobile client [1]. In addition, create and proposed mobile client cluster dependent on the thought of semantic direction similarity [1]. Besides, excavate the successive direction patterns of clients in a similar cluster dependent on their geographic highlights [1]. 
In the connected forecast module, in view of these semantic and geographic examples, build up a new cluster based forecasting strategy to anticipate a mobile client's next area [1]. This is the main work on foreseeing a cell phone client's next area by abusing both geographic and semantic highlights of directions [1]. Concluded a trial assessment, demonstrate the deliberate area forecast approach conveys magnificent execution.

\section{CONTRIBUTIONS}

The proposed structure Semantic forecast is another methodology for cell phone client's motion conduct mining and expectation. The issues and thoughts in Semantic forecast have not been investigated already in the exploration network. To create data mining algorithms to find semantic direction designs for mobile clients and direction designs for groups of comparative clients. Utilize the thought of semantic direction similitude, proposed to group comparative clients together. To create record structures dependent on prefix tree to speak to semantic direction designs in a conservative structure so as to encourage proficient expectation calculation. Utilize a genuine dataset, specifically, MIT reality dataset [1], in a progression of analyses to assess the exhibition of proposition. The outcomes show better execution in excess of other area forecasting systems as far as recall and precision.

\section{LITERATURE STUDY}

There are number of studies talked about the forecasting the mobile users next locations. Basically two methodologies are frequently used for solving this issue, they are Personal-based prediction and general-based prediction. The individual based expectation approach considers mobile client movement conduct of every person as autonomous and in this manner utilizes just the developments of an individual client to foresee their next area. Despite what might be expected, the general-based forecast makes a forecast dependent on the basic mobile client conduct of general cell phone clients.

A novel example, called Individual Life Pattern, which is mined association rules to construct movement forecast, and they uses such example to depict and show the cell phone clients' intermittent practices. To mine the development examples of a distinct client frame affiliation guidelines and utilize the standards to create area expectation. Furthermore, deliberate help then trust happening choosing affiliation directions for constructing expectations. A creative methodology proposed by Jeung et al, it envisages prospect positions of a mobile client through joining ordained movement capacities that is linear or non-direct representations, grasp point motions by way of advanced scientific recipes, with the development examples of the client, extricated by a changed form of the Apriori algorithms.

In current trend, various investigations on semantic direction data mining have showed up in the literature study [1].To investigate the geographic semantic data to mine semantic direction designs from cell phone clients' movement records proposed by Alvares et al [2].Initially find the stops of every direction and guide those stops to semantic spots to change geographic directions into semantic directions [1]. As a result of pertain a sequential pattern mining [2] calculation on semantic directions, and acquire regular patterns, to be specific, semantic direction designs, to speak to the regular semantic practices of cell phone clients.

The GPS heading also mishandles the cell bearing to deduce the semantic resemblance between two versatile customers. The cell course contains a progression of spatio common concentrations in kind of cell station ID, show up time, and leave time [1]. A new method named closeness estimation, specifically, Maximal Semantic Trajectory Pattern Similarity [2] to survey customer resemblance. In this way, the likeness of two PDA customers, paying little respect to whether sentient in diverse urban networks, might be surveyed reliant on the near semantic heading plans.

\section{OVERVIEW}

The idea of semantic direction a new area movement forecast structure, specifically, Semantic Predict, in view of together the geographic and semantic highlights in directions. The projected move toward work for areas where the clients may never visit, for example an area in different urban communities. The Semantic Predict structure comprises of two modules that is and own preparing part, and an connected expectation module.

\section{PREPROCESSING TECHNIQUES}

The thought is to investigate the exercises of cell phone clients, caught in semantic directions, to improve exactness of predict the location [2]. The training module incorporates three stages. The initial step, named data preprocessing, changes every client's directions as stay point arrangements. The subsequent advance, named semantic mining, extricates clients' semantic practices [2]. It additionally acquires client bunches dependent on the semantic conduct comparability of clients. The final step, named geographic mining, removes the geographic practices of clients in each group. We propose a scoring capacity to assess the likelihood for a point to be the next point [2]. In this regard think about geographic data as well as semantic data. In the initial stage, figure the geographic score and determine a few applicant ways. At that point, the semantic score of every applicant way is assessed [2]. At long last, process a weighted normal of geographic score and semantic score for every competitor way to choose the most likely way for anticipating the following area in a client's turn. To the information preprocessing step changes every client's GPS directions into stay area groupings [2].

This structure can manage both the GPS directions and cell directions [2]. For GPS direction, pursue to find stay focuses from clients' GPS directions using Zheng et al's Work. For cell directions use Ying et al's approach which regards a cell as a geographic area [1].

The stay time in a cell is inferred by figuring the contrast between the times a client lands in and leaves the phone [1]. A client indicated time edge is utilized to channel the cells with stay time shorter than the edge [1]. 
The rest of the cells are additionally separated by the quantity of clients went through. At last, the stay areas are acquired and every direction is changed into a stay areas arrangement [1].

\section{EVALUATIONS}

In this area, direct a progression of investigations to assess the exhibition for the projected location forecast system utilizing the MIT reality mining dataset[2]. First of all present the information arrangement on the MIT reality mining dataset and afterward present the assessment approach [1]. At last, present our exploratory outcomes pursued by discourses [1].

The MIT reality mining dataset is a cell phone dataset gathered by MIT Media Laboratory from 2004 to 2005[1]. The dataset contains 106 cell phone clients more than 500,000 hours of consistent every day exercises [1]. As appeared, the stay time in a cell can be determined by ascertaining the distinction in timestamp when a client lands in and leaves the cell. Along these lines, we can without much of a stretch find the stay cells of every cell direction [1].

While this dataset contains client commented on cell names, they innately are semantic directions as appeared in the Figure 1 [1]. Be that as it may, the explanation terms are differing. For instance, one may clarify a cell as "MLAB" while another person may comment on it as "Media Laboratory", despite the fact that it's clearly that this cell is MIT Media Laboratory. Also, numerous terms are geographic terms, for example, "Park zone" [1]. In spite of the fact that we try a great deal of endeavors to make sense of the semantics of the explanation terms in the log, there are shockingly still a few terms which we can't make certain of their implications. As a result, stem such term as "ambiguous"[1].

Among the 106 cell phone clients, there are seven clients who don't have cell direction logs, and 10 clients who don't have cell explanation logs [1]. Subsequently, in the wake of discarding these clients, information from the staying 89 cell phone clients are utilized in our tests [1]. For every cell phone client, we arbitrarily select 80 percent of their cell directions as the preparation dataset [1]. The rest of the direction structure is the testing dataset. At that point, we utilize the preparation dataset to acquire semantic direction design tree for every portable client, and stay area design tree for every

$$
\begin{aligned}
\text { precision } & =\frac{T P}{T P+F P} \\
\text { recall } & =\frac{T P}{T P+F N} \\
F 1 & =\frac{2 \times \text { precision } \times \text { recall }}{\text { precision }+ \text { recall }} \\
\text { accuracy } & =\frac{T P+T N}{T P+F N+T N+F P} \\
\text { specificity } & =\frac{T N}{T N+F P}
\end{aligned}
$$

client bunch. At last, we use Equation to assess the score of next area of every direction in testing dataset dependent on their semantic direction design tree and stay area design tree [1].

\section{A..Assessment Methodology}

The below things are the essential estimations for the exploratory evaluation. The Precision, Recall, and F-measure are described as underneath Equations. Here TP and TN show the quantity of right forecasts and inaccurate expectations, separately. Also, we utilize the normal development rate to quantify the rate our proposed technique beats different strategies.

The investigations are isolated into two sections they are sensitivity tests and framework assessment. The sensitivity tests assesses the projected strategies inside the semantic foresee system under different parameter settings. In this examination study, guarantee that the semantic data is a basic factor for location forecast and incomplete coordinating procedure could improve the review of expectation.

\section{B. Sensitivity Tests}

These tests assess methodology under different parameter settings as far as exactness .As appeared in Figure 17, the accuracy of our strategy is improved after is expanded, that is high exactness is accomplished once give more weight in late portable moves. It approves suspicion that later portable moves conceivably greatly affect anticipating the following move. In any case, likewise see that the improvement isn't huge meanwhile receive a halfway coordinating procedure. As a next move result, the obsolete portable move might be once in a while coordinated with an example. Likewise can see that the accuracy decays as increments, that is, as more weight is doled out to semantic score, the exactness gets lower. Repudiates presumption that semantic data improves the forecast accept this is on the grounds that the groups of clients bunches with comparable semantic practices.
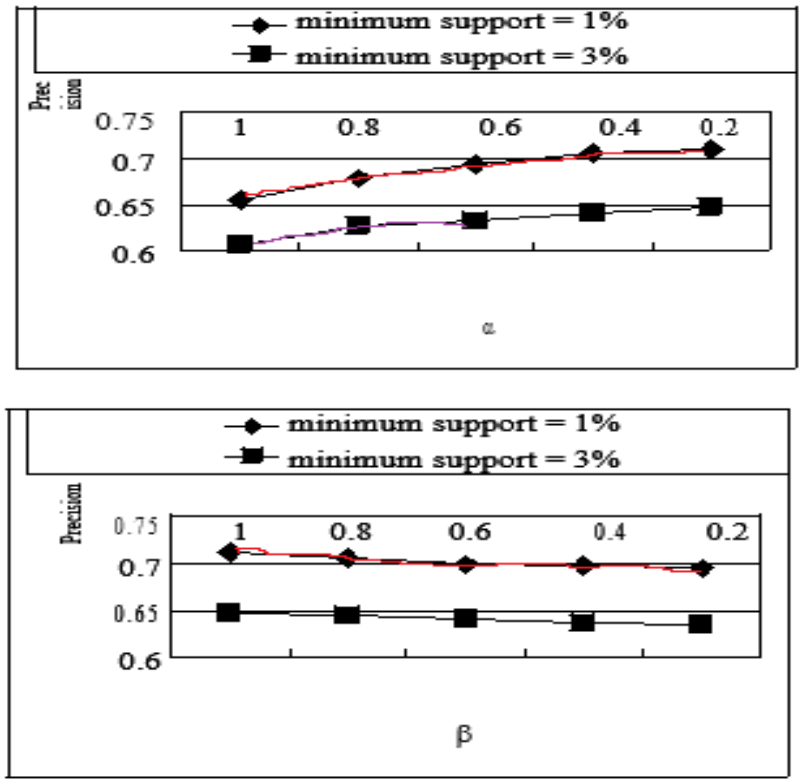

Figure: 1 parameter settings for Precision

At that point, assess the effect of the semantic bunching expectation model.Figure2 we can see that out performs none-grouping approach as far as the exactness, Recall and F-measure tactic through body-grouping tactic is $21.07 \%$ for accuracy, 25.05\% for review, 23.63\% for F-measure, correspondingly. 


\section{Prediction of Cell Phone Client's Location utilizing Semantic Trajectory}

This exhibits the semantic bunching procedure is viable in refining the projected expectation structure. Likewise can see that this methodology is steadier than then one-bunching approach, on the grounds that the grouping step bunches comparable clients with the end goal that most examples, found for each bunch don't vary.

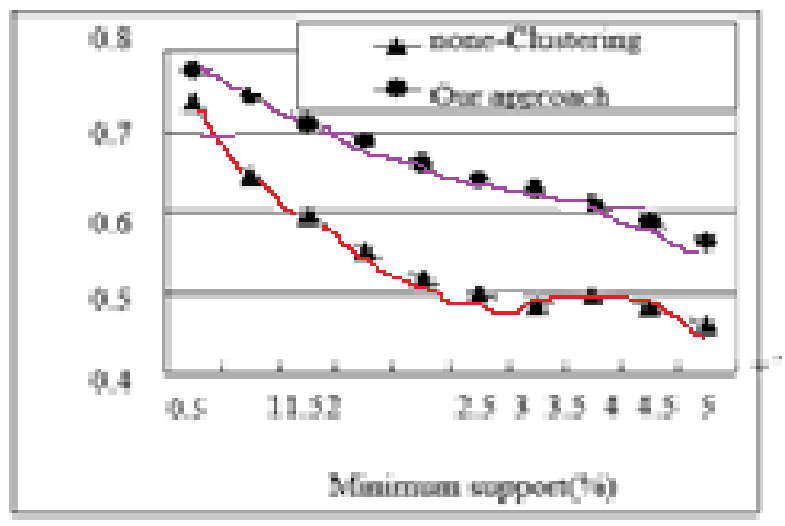

Figure: 2 semantic clustering impression

\section{CORRELATION OF FORECAST TACTICS}

The investigation dissects the precision, recall and F-measure of inspected forecasting systems [1]. Figure 3 indicates that semantic prediction isn't superior to FM as far as accuracy, however fundamentally beats it as far as recall and F-measure on the grounds that semantic foresee utilizes the fractional coordinating methodology. It additionally leads semantic prediction certain client travels are not unsurprising by different methods. In actuality, FM predicts a cell phones move just if their ongoing track is a sub arrangement of the prefix of certain examples. The normal improvement paces of semantic anticipate over FM are 216.53\% for review, 94.35\% for F-Measure, separately [1].

Likewise see that semantic foresee is marginally superior to GO regarding exactness, yet essentially beats it as far as review and F-measure, in light of the fact that semantic anticipate considers the semantic bunching as well as the semantic score of the following area of client's movements in the location forecast. Since the grouping step depends on client's semantic similitude's, the review of semantic forecast can accomplish 90\%. The normal improvement paces of semantic expectation concluded are $21.01 \%$ for precision, $26.057 \%$ for recall, and $23.56 \%$ for F-measure, individually.

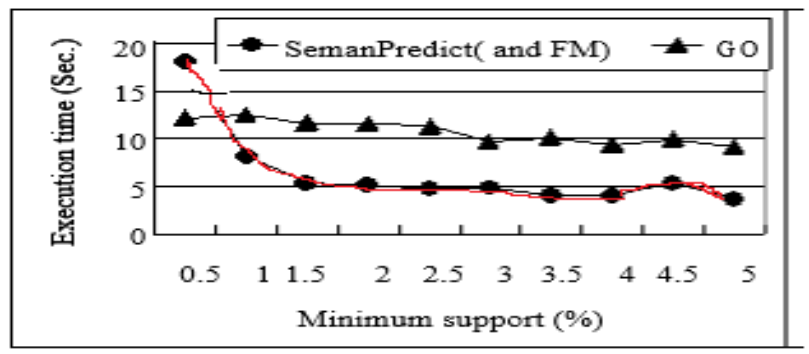

Figure.3 Semantic prediction Training

\section{EFFICIENCY EVALUATION}

Additionally lead investigations to assess the effectiveness of this methodology and other forecast methodologies under different least backings in disconnected preparing module and online expectation module, separately. In spite of the fact that this methodology needs to manage the semantic mining in disconnected preparing module, the semantic bunching has assembled all clients' direction signs into little sets [1]. Consequently, the execution time for geographic mining in our methodology is fundamentally not as much as that in GO. Likewise see that the execution time of expectation in this methodology is longer than that in GO and FM [1]. The explanation is that our methodology needs to ascertain the Semantic Score of every applicant way, yet GO doesn't have this overhead. In spite of the fact that FM likewise needs to compute the Semantic Score, full-coordinating innately prompts less applicant ways. Nonetheless, it is sensible that the execution time of our methodology is constrained by seconds in online forecast module [1].

\section{CONCLUSIONS}

In this research, recommend a new structure, by investigating the semantic directions of cell phone clients, to anticipate the next location of a cell phone client on the side of different location based administrations. The center of this structure is a fresh forecast methodology it assesses the notch of subsequent top over area aimed at assumed cell phone client. The structure of Semantic Prediction, suggest a fresh cluster based forecast procedure to foresee the following area of a portable client. It is the foremost effort that adventures together semantic and geographic data in directions aimed at area expectation. From side to side a progression of trials, approve this proposition also demonstrate the projected area expectation structure was fantastic execution beneath different circumstances. With respect to the forthcoming work, intend to plan further developed expectation techniques to improve the nature of area forecasts in area based administrations.

\section{REFERENCES}

1. cs.uef.fi

2. Josh Jia-Ching Ying, Wang-Chien Lee, Tz-Chiao Weng, Vincent S. Tseng. "Semantictrajectory mining for location prediction",Proceedings of the 19th ACM SIGSPATIALInternationa Conference on Advances inGeographic Information Systems - GIS '11,2011.

3. Jia-Ching Ying, Huan-Sheng Chen, Kawuu W.Lin, Eric Hsueh-Chan $\mathrm{Lu}$ et al. "Semantictrajectory-based high utility itemrecommendation system", Expert Systems withApplications, 2014.

4. Carmela Comito. "Where are You Going? NextPlace Prediction from Twitter", 2017 IEEEInternational Conference on Data Science andAdvanced Analytics (DSAA), 2017.

5. J. J.-C. Ying, E. H.-C. Lu, W.-C. Lee, T.-C. Weng, V. S. Tseng. Mining User Similarity from Semantic Trajectories. In Proceedings of ACM SIGSPATIAL International Workshop on Location Based Socia Networks (LBSN' 10), San Jose, California, USA, November 2, 2010.

6. Y. Zheng, L. Zhang, and X. Xie. Recommending friends and locations based on individual location history. ACM Transaction on the Web, 2010.

7. Yu Zheng, Lizhu Zhang, Xing Xie, Wei-Ying Ma. Mining interesting locations and travel sequences from GPS trajectories. WWW, 2009

8. Yu Zheng, Lizhu Zhang, Xing Xie, Wei-Ying Ma. Mining Correlation Between Locations Using Human Location History. ACM SIGSPATIAL GIS, 2009.

9. J. Liu, O. Wolfson, H. Yin. Extracting Semantic Location from Outdoor Positioning Systems. MDM, 2006

10. E. H.-C. Lu and V. S. Tseng. Mining Cluster-Based Mobile Sequentia Patterns in Location-Based Service Environments. MDM, 2009.

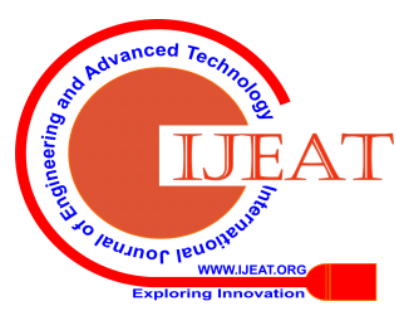


11. M. Morzy. Prediction of moving object location based on frequent trajectories. ISCIS, volume 4263 of LNCS, pages 583-592. Springer, 2006.

12. M. Morzy. Mining frequent trajectories of moving objects for location prediction. MLDM, volume 4571 of LNCS, pages 667-680. Springer, 2007.

13. Monreale, F. Pinelli, R. Trasarti, F. Giannotti. WhereNext: a location predictor on trajectory pattern mining. KDD 2009: 637-646.

14. L. O. Alvares, V. Bogorny, A. Palma, B. Kuijpers, B. Moelans, and J. A. F. Macedo. Towards Semantic Trajectory Knowledge Discovery. Technical Report, Hasselt University, Belgium, Oct. 2007.

15. Bogorny, B. Kuijpers, and L. O. Alvares. ST-DMQL: A Semantic Trajectory Data Mining Query Language. International Journal of Geographical Information Science, Vol. 23, No. 10, 1245-1276, Oct. 2009

16. N. Eagle, A. Pentland, and D. Lazer. Inferring Social Network Structure using Mobile Phone Data. In proceedings of the National Academy of Sciences (PNAS),106(36), pp. 15274-15278, 2009.

17. H. Jeung, Q. Liu, H. T. Shen, and X. Zhou. A hybrid prediction model for moving objects. ICDE 2008: 70-79.

18. J. Pei, J. Han, B. Mortazavi-Asl, H. Pinto, Q. Chen, U. Dayal, and M.C. Hsu. PrefixSpan: Mining sequential patterns efficiently by prefix-projected pattern growth. In Proceedings of the 17th International Conference on Data Engineering (ICDE), 2001, 215-224.

19. G. Yavas, D. Katsaros, Ö. Ulusoy, and Y. Manolopoulos. A data mining approach for location prediction in mobile environments. D.K.E., 54(2):121-146, 2005.

20. Yang Ye*, Yu Zheng, Yukun Chen, Jianhua Feng, Xing Xie. Mining Individual Life Pattern Based on Location History. In proceedings of the International Conference on Mobile Data Management 2009 (MDM 2009). IEEE, 1-10.

21. S.-J. Yen, Y.-S. Lee, C.-H. Lin and J.-C. Ying, Investigating the Effect of Sampling Methods for Imbalanced Data Distributions, Proceedings of IEEE International Conference on Systems, Man, and Cybernetics (SMC'2006), pp. 4163-1468, October 2006. 